\title{
Pacific
}

Journal of

Mathematics

\section{TENSOR PRODUCTS OF STRUCTURES WITH INTERPOLATION}

\author{
FRIEDRICH WEHRUNG
}




\title{
TENSOR PRODUCTS OF STRUCTURES WITH INTERPOLATION
}

\author{
FRIEDRICH WEHRUNG
}

\begin{abstract}
While it is known that the tensor product of two dimension groups is a dimension group, the corresponding problem for interpolation groups has been open for a while. We solve this problem here, by proving that the tensor product of two interpolation groups may not be an interpolation group, even for directed, torsion-free interpolation groups. We also solve the corresponding problems for refinement monoids (with tensor product of commutative monoids) and for lattice-ordered groups (with tensor product of partially ordered abelian groups).
\end{abstract}

\section{Introduction.}

Let $A$ and $B$ be two partially ordered abelian groups. Then the tensor product $A \otimes B$ (in the category of $\mathbb{Z}$-modules) can be given a structure of partially ordered abelian group, with positive cone the set of all sums $\sum_{i<n} a_{i} \otimes b_{i}$ where $n \in \mathbb{N}$ and for all $i<n,\left(a_{i}, b_{i}\right) \in A^{+} \times B^{+}$(this tensor product is related but not isomorphic to either kind of tensor product $A \otimes_{o} B$ or $A \otimes_{\ell} B$ considered in [9], where the result is always forced into being a $\ell$-group even for arbitrary partially ordered abelian groups $A$ and $B$ ). It is proven in [5] that the tensor product of two dimension groups (i.e. directed, unperforated partially ordered abelian groups with the interpolation property) is a dimension group. Then K.R. Goodearl asks in [6, Question 26] whether this holds for interpolation groups, i.e. whether the tensor product of two interpolation groups is an interpolation group.

We answer this question here, by giving several counterexamples where this does not hold (Examples 1.3 to 1.5), each of them with a specific feature. Our search for those counterexamples leads us first to study the connection between the positive cone of the tensor product of two partially ordered abelian groups and the tensor product of their positive cones as cancellative commutative monoids. Indeed, Example 1.3 shows that both are not necessarily isomorphic. Our constructions turn out in fact to be related to a counterexample of Manfred Dugas to [6, Question 2]. The common pattern between these counterexamples is that they show in particular that tensor 
product of interpolation groups does not preserve 'orthogonality' (as defined at the end of the introduction): $a_{0}$ may be orthogonal to $a_{1}$ without $a_{0} \otimes b$ being orthogonal to $a_{1} \otimes b(b \geq 0)$. This quite irregular behavior does not happen for [conical] commutative monoids (Corollary 2.11) or for two interpolation groups one of which is unperforated (Corollary 2.12). Finally, still on the negative side, we show in Example 1.6 that the tensor product of two lattice-ordered groups may not be lattice-ordered - thus confirming that even for lattice-ordered $A$ and $B, A \otimes B$ may not be isomorphic to Martinez' $A \otimes_{o} B$.

Nevertheless, it turns out that in many natural contexts, analogues of Goodearl's question find positive answers: this fact is mainly supported by our following Theorem 2.9, which states that the tensor product of two refinement monoids (in the category of commutative monoids) is a refinement monoid. Towards this goal, we prove in fact that the tensor product of two conical refinement monoids is a conical refinement monoid (Theorem 2.7). The proof of these two results uses a "half-syntax, half-semantic" method, where elements of the tensor product $A \otimes B$ are viewed as [equivalence classes of] words on the alphabet $A \times B$, on which we apply a certain rewriting rule (relevant only when $A$ and $B$ are refinement monoids). We also refer for example to $[\mathbf{7}, \mathbf{8}]$ for work about tensor products of semigroups (but preservation of the finite refinement property under tensor product is not stated there). Other positive results of preservation by tensor products may be found in $[3,9]$ for vector lattices (with various sorts of tensor products, all different from those considered in this article!) and of course [5] for dimension groups.

We will mainly follow the notations and terminology of $[\mathbf{5}, \mathbf{6}, \mathbf{1 1}]$. In particular, $\mathbb{N}=\mathbb{Z}^{+} \backslash\{0\}=\{1,2,3, \ldots\}$. If $a_{0}, \ldots, a_{m-1}, b_{0}, \ldots, b_{n-1}$ are elements of a given preordered set, then we will write $a_{0}, \ldots, a_{m-1} \leq b_{0}, \ldots$, $b_{n-1}$ instead of $(\forall i<m)(\forall j<n)\left(a_{i} \leq b_{j}\right)$. For every commutative monoid $M, \operatorname{Grot}(M)$ will denote the Grothendieck group (also called 'universal group') of $M$; it is a partially preordered abelian group, with positive cone the image of $M$. If $M$ is a commutative monoid, then we will denote by $\leq_{\text {alg }}$ the algebraic preordering of $M$, defined by $x \leq_{\text {alg }} y \Leftrightarrow(\exists z)(x+z=y)$. We will say that $M$ is conical when it satisfies $(\forall x, y)(x+y=0 \Rightarrow x=y=0)$ (this concept has been given various names in the literature besides 'conical', such as 'centerless', 'positive' or 'zerosumfree'). The finite refinement property is the following axiom:

$$
\begin{aligned}
\left(\forall_{i<2} a_{i}, b_{i}\right)\left(a_{0}+a_{1}\right. & =b_{0}+b_{1} \\
\quad & \left.\Rightarrow\left(\exists_{i, j<2} c_{i j}\right)(\forall i<2)\left(a_{i}=c_{i 0}+c_{i 1} \text { and } b_{i}=c_{0 i}+c_{1 i}\right)\right)
\end{aligned}
$$


while the interpolation property is the axiom

$$
\left(\forall_{i<2} a_{i}, b_{i}\right)\left(a_{0}, a_{1} \leq b_{0}, b_{1} \Rightarrow(\exists c)\left(a_{0}, a_{1} \leq c \leq b_{0}, b_{1}\right)\right) .
$$

It is well-known [6, Proposition 2.1] that if $G$ is a partially ordered abelian group, then $G$ satisfies the interpolation property if and only if $G^{+}$satisfies the finite refinement property. In general, a refinement monoid is a commutative monoid satisfying the finite refinement property. An interpolation group is a (non necessarily directed) partially ordered abelian group satisfying the interpolation property. If $M$ is an ordered monoid, two positive elements $a$ and $b$ of $M$ are said to be orthogonal, in notation $a \perp_{M} b$, when $M$ satisfies $(\forall x)(x \leq a, b \Rightarrow x \leq 0)$; note that if $G$ is a partially ordered abelian group, then, for elements of $G^{+}$, orthogonality in $G^{+}$(algebraically ordered) is weaker than orthogonality in $G$, and if $G$ is an interpolation group, then both notions coincide. A partially ordered abelian group is unperforated when for all $m \in \mathbb{N}$, it satisfies the statement $(\forall x)(m x \geq 0 \Rightarrow x \geq 0)$.

\section{Generalities about tensor products. Case of interpolation groups.}

The structures that we shall consider in this paper will always be sets endowed with a commutative, associative operation + with unit element 0 (commutative monoids, abelian groups), and possibly with a partial ordering $\leq$ (partially ordered abelian groups). If $A, B, C$ are structures and $f: A \times B \rightarrow C$, say that $f$ is a bimorphism when for all $a \in A$ (resp. $b \in B$ ), the map $f\left(a,,_{-}\right)$(resp. $\left.f(-, b)\right)$ is a homomorphism of monoids; if in addition $\leq$ is in the language, say that $f$ is positive when for all positive $a \in A$ and $b \in B$, we have $f(a, b) \geq 0$. We shall say that the [positive] bimorphism $f$ is universal (relatively to a given category of structures) when for every structure $D$ and every [positive] bimorphism $g: A \times B \rightarrow D$, there exists a unique [positive] homomorphism $\bar{g}: C \rightarrow D$ such that $\bar{g} \circ f=g$; in this case the pair $(C, f)$ is unique up to isomorphism and the custom is to call it the tensor product of $A$ and $B$, and to write $C=A \otimes B, f(a, b)=a \otimes b$. In fact this notion is very sensitive to the category of structures under consideration, thus the latter will most of the time be used as a superscript: thus $\otimes^{\mathrm{cm}}$ will denote the tensor product of commutative monoids, $\otimes^{\mathrm{ag}}$ will denote the tensor product of abelian groups and $\otimes^{\text {oag }}$ will denote the tensor product of partially ordered abelian groups. For all the three categories above, the tensor product always exists and this is in fact, in an abstract model-theoretical setting, a general feature of the models of first-order theories whose axioms are universal Horn sentences - but observe that the case of partially ordered abelian groups would not completely fit in this general framework: in 
the above definition of a positive bimorphism, $f(a,-)$ may not be a positive homomorphism if $a \nsupseteq 0$.

Proposition 1.1. Let $A$ and $B$ be partially ordered abelian groups. Then the tensor product $A \otimes^{\mathrm{ag}} B$ [of the underlying abelian groups of $A$ and $B$ ] can be given a structure of partially ordered abelian group with positive cone the set of all finite sums of pure tensors of the form $a \otimes^{\mathrm{ag}} b$ where $(a, b) \in$ $A^{+} \times B^{+}$, and this partially ordered abelian group is in fact a tensor product of $A$ and $B$ in both the category of preordered abelian groups and the category of ordered abelian groups.

Proof. It is obvious that the construction above yields a partially preordered abelian group. That it yields in fact a partially ordered abelian group is not trivial, but it is proved in [5, Proposition 2.1]. It is also straightforward to verify that $(a, b) \mapsto a \otimes^{\mathrm{ag}} b$ is a universal positive bimorphism for preordered abelian groups, thus a fortiori for ordered abelian groups.

There are also connections between $\otimes^{\text {oag }}$ and $\otimes^{\mathrm{cm}}$. For every partially ordered abelian group $A$, put $A^{ \pm}=A^{+}+\left(-A^{+}\right)$("directed part" of $A$ ).

Proposition 1.2. Let $A$ and $B$ be partially ordered abelian groups. Then the following holds:

(a) If both $A$ and $B$ are directed, then $A \otimes^{\mathrm{oag}} B \cong \operatorname{Grot}\left(A^{+} \otimes^{\mathrm{cm}} B^{+}\right)$.

(b) Suppose that one of the following conditions holds:

(i) Both $A$ and $B$ are directed;

(ii) Both $A^{ \pm}$and $B^{ \pm}$are torsion-free, and either $A$ or $B$ is torsionfree;

(iii) Either $A$ or $B$ is unperforated.

Then $\left(A \otimes^{\text {oag }} B\right)^{+} \cong\left(A^{ \pm} \otimes^{\text {oag }} B^{ \pm}\right)^{+}$.

Proof. (a) Let $C=\operatorname{Grot}\left(A^{+} \otimes^{\mathrm{cm}} B^{+}\right)$, let $c \mapsto[c]$ the natural map from $A^{+} \otimes^{\mathrm{cm}} B^{+}$to $C$ (its range is $C^{+}$). Note that $C$ is a preordered abelian group. Let $\odot$ be the map from $A^{+} \times B^{+}$to $C^{+}$defined by $a \odot b=\left[a \otimes^{\mathrm{cm}} b\right]$. Since $\otimes^{\mathrm{cm}}$ is a bimorphism of monoids and both $A$ and $B$ are directed, $\odot$ can be extended to a unique positive bimorphism from $A \times B$ to $C$, that we shall still denote by $\odot$. Then it is routine to verify that this extended $\odot$ is a universal positive bimorphism of preordered abelian groups. Therefore, by Proposition 1.1, $C$ is in fact an ordered abelian group and it is isomorphic to $A \otimes^{\text {oag }} B$.

(b) There is nothing to prove in case (i). In general, for every partially ordered abelian group $C$, denote by $j_{C}$ the inclusion map from $C^{ \pm}$into $C$. In case (ii), suppose for example that $B$ is torsion-free. Then both positive 
homomorphisms $\operatorname{id}_{A^{ \pm}} \otimes^{\mathrm{ag}} j_{B}: A^{ \pm} \otimes^{\mathrm{ag}} B^{ \pm} \rightarrow A^{ \pm} \otimes^{\mathrm{ag}} B$ and $j_{A} \otimes^{\mathrm{ag}} \mathrm{id}_{B}:$ $A^{ \pm} \otimes^{\mathrm{ag}} B \rightarrow A \otimes^{\mathrm{ag}} B$ are in fact one-to-one (because $A^{ \pm}$and $B$ are torsionfree abelian groups, thus [4, vol. I, Theorem 60.6] flat abelian groups), thus their composition $j_{A} \otimes^{\mathrm{ag}} j_{B}: A^{ \pm} \otimes^{\mathrm{ag}} B^{ \pm} \rightarrow A \otimes^{\mathrm{ag}} B$ is also one-to-one. Since $\left(A \otimes^{\mathrm{oag}} B\right)^{+}=j_{A} \otimes^{\mathrm{ag}} j_{B}\left[\left(A^{ \pm} \otimes^{\mathrm{oag}} B^{ \pm}\right)^{+}\right]$, the conclusion follows in case (ii).

In case (iii), suppose for example that $A$ is unperforated. We first prove that $A^{ \pm}$is a pure subgroup of $A$, i.e. for all $m \in \mathbb{N}, m A \cap A^{ \pm}=m A^{ \pm}$. Thus let $a \in A$ such that $m a \in A^{ \pm}$. By definition, there are $b$ and $c$ in $A^{+}$ such that $m a=b-c$. It follows that $m a \leq b \leq m b$, whence, since $A$ is unperforated, $a \leq b$. Therefore, $a=b-(b-a)$ belongs to $A^{ \pm}$. It follows $\left[4\right.$, vol. I, theorem 60.4] that $j_{A} \otimes^{\mathrm{ag}} \mathrm{id}_{B}: A^{ \pm} \otimes^{\mathrm{ag}} B \rightarrow A \otimes^{\mathrm{ag}} B$ is one-to-one. Furthermore, $A^{ \pm}$is unperforated, thus torsion-free, thus it is a flat abelian group, thus $\operatorname{id}_{A^{ \pm}} \otimes^{\mathrm{ag}} j_{B}: A^{ \pm} \otimes^{\mathrm{ag}} B^{ \pm} \rightarrow A^{ \pm} \otimes^{\mathrm{ag}} B$ is one-to-one. Therefore, $j_{A} \otimes^{\mathrm{ag}} j_{B}: A^{ \pm} \otimes^{\mathrm{ag}} B^{ \pm} \rightarrow A \otimes^{\mathrm{ag}} B$ is one-to-one, and we conclude as at the end of case (ii).

The following counterexample shows that in the hypothesis of Proposition 1.2 , (b, iii), one cannot weaken "unperforated" into "torsion-free". It also solves (negatively) [6, Problem 26].

Example 1.3. Two interpolation groups $A$ and $B$ such that $A$ is torsionfree, $B$ is directed and $A \otimes^{\text {oag }} B$ is not an interpolation group, although $A^{ \pm} \otimes^{\text {oag }} B^{ \pm}=A^{ \pm} \otimes^{\text {oag }} B$ is an interpolation group.

Proof. Let $A=\{(m, n) \in \mathbb{Z} \times \mathbb{Z}: m \equiv n(\bmod 2)\}$, endowed with the positive cone $A^{+}=2 \mathbb{Z}^{+} \times 2 \mathbb{Z}^{+}$. Since $A^{+}$satisfies interpolation, $A$ satisfies interpolation; note also that $A$ is torsion-free, but not directed. Let $B=$ $\mathbb{Q} \times_{\text {lex }} \mathbb{Z} / 2 \mathbb{Z}$ be the lexicographic product of $\mathbb{Q}$ (with the natural ordering) and $\mathbb{Z} / 2 \mathbb{Z}$ (with the discrete ordering). By [6, Corollary 2.12], $B$ is an interpolation group. It is clearly directed. For every $r \in \mathbb{Q}$, identify $r$ with $(r, 0) \in B$, and put $\varepsilon=(0,1)$. Thus every element of $B$ can be written $r+k \varepsilon$ with $r \in \mathbb{Q}$ and $k \in\{0,1\}$ (note that $2 \varepsilon=0$ ).

Now, let $\bar{A}$ be the abelian group $\mathbb{Q} \times \mathbb{Q}$. Then every element of $\bar{A}$ can be written $(1 / q) a$ where $q \in \mathbb{N}$ and $a \in A$. Let $C=\bar{A} \times(A / 2 A)$, and let $P$ be defined by

$$
P=\left\{((1 / q) a, b+2 A) \in C: 0 \leq_{A} b \leq_{A} a\right\} .
$$

It is easy to verify that $P$ is the positive cone of a structure of partially ordered abelian group on $C$. Then let $\odot$ be the map from $A \times B$ to $C$ defined by

$$
a \odot(r+k \varepsilon)=(r a, k a+2 A) \quad(\text { all } a \in A, r \in \mathbb{Q} \text { and } k \in\{0,1\}) .
$$

Then it is easy to verify that $\odot$ is a positive bimorphism from $A \times B$ to $C$ (note: in fact, $\odot$ is universal, but we will not need this). Thus there 
exists a positive homomorphism $\phi$ from $A \otimes^{\text {oag }} B$ to $C$ such that for all $(a, b) \in A^{+} \times B^{+}$, one has $a \odot b=\phi\left(a \otimes^{\text {oag }} b\right)$.

Now consider the following three elements of $A$ :

$$
a=(2,0) ; \quad a^{\prime}=(0,2) ; \quad c=(-1,1) .
$$

Thus $a, a^{\prime} \in A^{+}, a \perp_{A} a^{\prime}$ and $a+2 c=a^{\prime}$, although $a \notin 2 A$. Then $a^{\prime} \otimes^{\text {oag }} \varepsilon=a \otimes^{\text {oag }} \varepsilon+2 c \otimes^{\text {oag }} \varepsilon=a \otimes^{\text {oag }} \varepsilon+c \otimes^{\text {oag }} 2 \varepsilon=a \otimes^{\text {oag }} \varepsilon$ (because $2 \varepsilon=0)$, thus we get easily that $0, a \otimes^{\text {oag }} \varepsilon \leq a \otimes^{\text {oag }}(1+\varepsilon), a^{\prime} \otimes^{\text {oag }}(1+\varepsilon)$. But suppose that there exists $z \in A \otimes^{\text {oag }} B$ such that $0, a \otimes^{\text {oag }} \varepsilon \leq z \leq$ $a \otimes^{\text {oag }}(1+\varepsilon), a^{\prime} \otimes^{\text {oag }}(1+\varepsilon)$. Taking the image of these inequalities under $\phi$ yields $(0,2 A),(0, a+2 A) \leq_{C} \phi(z) \leq_{C}(a, a+2 A),\left(a^{\prime}, a^{\prime}+2 A\right)$. Since $a \perp_{A} a^{\prime}$, we necessarily have $\phi(z)=(0, \beta)$ for some $\beta \in A / 2 A$. Since $(0,2 A) \leq_{C}(0, \beta)$, the definition of $\leq_{C}$ yields easily that there exists $b \in \beta$ such that $0 \leq \leq_{A} b \leq_{A} 0$, i.e. $\beta=2 A$. Thus $(0, a+2 A) \leq(0,2 A)$, thus, as before, $a \in 2 A$, a contradiction. This proves that $A \otimes^{\text {oag }} B$ does not satisfy the interpolation property.

However, $A^{ \pm}$is isomorphic to $\mathbb{Z} \times \mathbb{Z}$ with its natural ordering, thus $A^{ \pm} \otimes^{\text {oag }} B \cong B \times B$ is an interpolation group.

This example can easily be modified to find a counterexample to [6, Problem 26] even for directed interpolation groups:

Example 1.4. Two directed interpolation groups $A^{\prime}$ and $B$ such that $A^{\prime}$ is torsion-free and $A^{\prime} \otimes^{\text {oag }} B$ is not an interpolation group.

Proof. Let $A$ and $B$ be the two partially ordered abelian groups defined in the previous example, and let $A^{\prime}=\mathbb{Q} \times_{\text {lex }} A$ : thus, $A^{\prime}$ is a directed interpolation group [6, Corollary 2.12]. Replace the previous values of $a, a^{\prime}$ and $c$ by $(0, a),\left(0, a^{\prime}\right)$ and $(0, c)$. Then it is obvious that one has again $a+2 c=a^{\prime}$ and $a \perp_{A^{\prime}} a^{\prime}$ but $a \notin 2 A^{\prime}$. Then it is easy to modify the proof of previous example to verify that although $0, a \otimes^{\mathrm{oag}} \varepsilon \leq a \otimes^{\mathrm{oag}}(1+\varepsilon), a^{\prime} \otimes^{\mathrm{oag}}(1+\varepsilon)$, there exists no $z$ in $A^{\prime} \otimes^{\text {oag }} B$ such that $0, a \otimes^{\text {oag }} \varepsilon \leq z \leq a \otimes^{\text {oag }}(1+\varepsilon), a^{\prime} \otimes^{\text {oag }}(1+\varepsilon)$.

Note that in Example 1.4, $B$ is not torsion-free. However, in order to overcome this last difficulty, one has to find a more involved counterexample (experimentally, it is the result of Proposition 1.2, (b, ii) which makes things more difficult):

Example 1.5. Two torsion-free directed interpolation groups $A$ and $B$ such that $A \otimes^{\text {oag }} B$ is not an interpolation group.

Proof. Let $G=\{(x, y, z) \in \mathbb{Z} \times \mathbb{Z} \times \mathbb{Z}: x+y+z \equiv 0(\bmod 2)\}$, with positive cone $G^{+}=\{0\} \times 2 \mathbb{Z}^{+} \times 2 \mathbb{Z}^{+}$(note that indeed, $G^{+} \subseteq G$ ). Then $G$ is a 
torsion-free interpolation group, but it is not directed. Put $A=\mathbb{Q} \times_{\text {lex }} G$. Thus $A$ is a directed torsion-free interpolation group.

Now let $H=\mathbb{Z}$ be endowed with the positive cone $H^{+}=2 \mathbb{Z}^{+}$, and put $B=\mathbb{Q} \times_{\text {lex }} H$. Again, $H$ is a non-directed torsion-free interpolation group, and $B$ is a directed torsion-free interpolation group. By identifying, for every $r \in \mathbb{Q}, r$ with $(r, 0) \in B$ and by putting $\varepsilon=(0,1) \in B$, one sees again that every element of $B$ can be written in a unique way $r+k \varepsilon$ where $r \in \mathbb{Q}$ and $k \in \mathbb{Z}$. Note that $2 \varepsilon \in B^{+} \backslash\{0\}$ while $\varepsilon \notin B^{+}$.

Now, let $\bar{A}$ be the abelian group $\mathbb{Q} \times(\mathbb{Q} \times \mathbb{Q} \times \mathbb{Q})$, and let $C=\bar{A} \times A$ (considered as abelian group). Define the following subset of $C$ :

$$
\begin{aligned}
P=\left\{((1 / q) a, b): q \in \mathbb{N} \text { and } a \in A^{+}\right. & \text {and } b \in A \\
& \text { and } \left.\left(\exists x \in A^{+}\right)(-a \leq b-2 x \leq a)\right\} .
\end{aligned}
$$

It is clear that $P$ is the positive cone of a structure of partially ordered abelian group on $C$. Then let $\odot$ be the map from $A \times B$ to $C$ defined by

$$
a \odot(r+k \varepsilon)=(r a, k a) \quad(\text { all } a \in A, r \in \mathbb{Q} \text { and } k \in \mathbb{Z}) \text {. }
$$

It is easily checked that $\odot$ is a positive bimorphism. Thus there exists a unique positive homomorphism $\phi$ from $A \otimes^{\text {oag }} B$ to $C$ such that for all $(a, b) \in A \times B$, we have $\phi\left(a \otimes^{\text {oag }} b\right)=a \odot b$.

Now, define elements of $G$ by

$$
\begin{gathered}
\dot{a}_{0}=(0,2,0) ; \quad \dot{a}_{1}=(0,0,2) ; \\
\dot{a}=(2,0,0) ; \\
\dot{c}_{0}=(1,1,0) ; \quad \dot{c}_{1}=(1,0,1) .
\end{gathered}
$$

Then define elements of $A$ by

$$
a_{i}=\left(0, \dot{a}_{i}\right) \text { and } c_{i}=\left(1, \dot{c}_{i}\right) \quad(\text { all } i<2) ; \quad a=(2, \dot{a}) .
$$

For all $i<2$, since $\dot{a}_{i} \in G^{+}$, we have $a_{i} \in A^{+}$. By definition of the lexicographical product, we also have $c_{i} \in A^{+}$and $a \in A^{+}$. Furthermore, $\dot{a}+\dot{a}_{i}=2 \dot{c}_{i}$ thus $a+a_{i}=2 c_{i}$; thus $2 c_{i}-a=a_{i} \in A^{+}$. It is also immediate that $a_{0} \perp_{A} a_{1}$. Finally, since $(1,0,0) \notin G$, we have $\dot{a} \notin 2 G$, thus $a \notin 2 A$.

It follows that for all $i<2$, we have

$$
\begin{aligned}
a_{i} \otimes^{\text {oag }}(1+\varepsilon)+a \otimes^{\text {oag }} \varepsilon & =a_{i} \otimes^{\text {oag }} 1+\left(a_{i}+a\right) \otimes^{\text {oag }} \varepsilon \\
& =a_{i} \otimes^{\text {oag }} 1+2 c_{i} \otimes^{\text {oag }} \varepsilon \\
& =a_{i} \otimes^{\text {oag }} 1+c_{i} \otimes^{\text {oag }} 2 \varepsilon \\
& \in\left(A \otimes^{\text {oag }} B\right)^{+} .
\end{aligned}
$$


Therefore, we obtain the inequalities

$$
0,-a \otimes^{\text {oag }} \varepsilon \leq a_{0} \otimes^{\text {oag }}(1+\varepsilon), a_{1} \otimes^{\text {oag }}(1+\varepsilon) .
$$

Suppose that there exists $z \in A \otimes^{\text {oag }} B$ such that

$$
0,-a \otimes^{\text {oag }} \varepsilon \leq z \leq a_{0} \otimes^{\text {oag }}(1+\varepsilon), a_{1} \otimes^{\text {oag }}(1+\varepsilon) .
$$

Then, taking the image of these inequalities under $\phi$ yields

$$
\left(0_{\bar{A}}, 0_{A}\right),\left(0_{\bar{A}},-a\right) \leq_{C} \phi(z) \leq_{C}\left(a_{0}, a_{0}\right),\left(a_{1}, a_{1}\right) .
$$

Since $a_{0} \perp_{A} a_{1}$, it follows easily that there exists $b \in A$ such that $\phi(z)=$ $\left(0_{\bar{A}}, b\right)$. Since $\left(0_{\bar{A}}, 0\right) \leq_{C}\left(0_{\bar{A}}, b\right)$, we obtain $b \in 2 A^{+}$by definition of $P$. Similarly, since $\left(0_{\bar{A}},-a\right) \leq_{C}\left(0_{\bar{A}}, b\right)$, we obtain $a+b \in 2 A^{+}$. In particular, we obtain that $a \in 2 A$, a contradiction.

On the other hand, the tensor product of two dimension groups is a dimension group, as proved in [5, Proposition 2.3]. But even stronger structures may not be preserved any more under tensor product of partially ordered abelian groups! Here is an example with lattice-ordered groups:

Example 1.6. $\mathbb{R} \otimes^{\text {oag }} \mathbb{R}$ is not a lattice-ordered group.

Proof. We start with two claims.

Claim 1. Let $A$ and $B$ be torsion-free abelian groups and let $a, a^{\prime} \in A \backslash\{0\}$ and $b, b^{\prime} \in B \backslash\{0\}$ such that $a \otimes^{\mathrm{ag}} b=a^{\prime} \otimes^{\mathrm{ag}} b^{\prime}$. Then both $\left(a, a^{\prime}\right)$ and $\left(b, b^{\prime}\right)$ are not independent over $\mathbb{Z}$, i.e. there are non zero pairs of integers $\left(m, m^{\prime}\right)$ and $\left(n, n^{\prime}\right)$ such that $m a=m^{\prime} a^{\prime}$ and $n b=n^{\prime} b^{\prime}$.

Proof. Put $\bar{A}=\mathbb{Q} \otimes^{\text {ag }} A$ and $\bar{B}=\mathbb{Q} \otimes^{\text {ag }} B$. Since both $A$ and $B$ are torsionfree, both natural homomorphisms $A \rightarrow \bar{A}$ and $B \rightarrow \bar{B}$ are one-to-one; by torsion-freeness, the natural map from $A \otimes^{\mathrm{ag}} B$ to $\bar{A} \otimes^{\mathrm{ag}} \bar{B}$ is one-to-one [4, Theorem 60.6], thus one can identify $A \otimes^{\mathrm{ag}} B$ with its natural image into $\bar{A} \otimes^{\mathrm{ag}} \bar{B}$. Suppose now that $\left(a, a^{\prime}\right)$ is independent over $\mathbb{Z}$; thus it is also independent over $\mathbb{Q}$, thus (since $a \neq 0_{A}$ ) there exists an element $p$ of the algebraic dual $\bar{A}^{*}$ of the $\mathbb{Q}$-vector space $\bar{A}$ such that $p(a)=1$ but $p\left(a^{\prime}\right)=0$. Since $b \neq 0_{\bar{B}}$, there exists $q \in \bar{B}^{*}$ such that $q(b)=1$. But the equality $a \otimes^{\mathrm{ag}} b=a^{\prime} \otimes^{\mathrm{ag}} b^{\prime}$ implies that $p(a) q(b)=p\left(a^{\prime}\right) q\left(b^{\prime}\right)$, i.e. $1=0$, a contradiction.

Claim 2. For all $x \in \mathbb{Q} \backslash\{0\}$, one has $x \otimes^{\mathrm{ag}}(1 / x)=1 \otimes^{\mathrm{ag}} 1\left(\right.$ in $\left.\mathbb{R} \otimes^{\mathrm{ag}} \mathbb{R}\right)$. Proof. Write $x=p / q$ where $p \in \mathbb{Z} \backslash\{0\}$ and $q \in \mathbb{N}$. Then we have

$$
\begin{aligned}
x \otimes^{\mathrm{ag}}(1 / x)=(p / q) \otimes^{\mathrm{ag}}(q / p) & =p \cdot\left((1 / q) \otimes^{\mathrm{ag}}(q / p)\right)=(1 / q) \otimes^{\mathrm{ag}}(p \cdot(q / p)) \\
& =(1 / q) \otimes^{\mathrm{ag}} q=q \cdot\left((1 / q) \otimes^{\mathrm{ag}} 1\right)=1 \otimes^{\mathrm{ag}} 1 .
\end{aligned}
$$


Now, put $\alpha=\sqrt{2}$ (any positive irrational number would do). There are sequences $\left(p_{n}\right)_{n \in \mathrm{N}}$ and $\left(q_{n}\right)_{n \in \mathrm{N}}$ of elements of $\mathbb{N}$ such that $\lim _{n \rightarrow+\infty} p_{n} / q_{n}=\alpha$ and $(\forall n \in \mathbb{N})\left(p_{2 n} / q_{2 n}<\alpha<p_{2 n+1} / q_{2 n+1}\right)$. Put $\rho_{n}=p_{2 n+1} / q_{2 n+1}-p_{2 n} / q_{2 n}$. We may assume without loss of generality that for all $n \in \mathbb{N}$, we have $0 \leq \rho_{n} \leq 1 / n$ and $1 / 2 \leq p_{n} / q_{n} \leq 2$.

Now put $a=1 \otimes^{\text {oag }} 1$ and $b=\alpha \otimes^{\text {oag }}(1 / \alpha)$. Suppose that $\{a, b\}$ has a least upper bound in $\mathbb{R} \otimes^{\text {oag }} \mathbb{R}$; put $c=2(a \vee b)-(a+b)$. Thus $c=$ $(a \vee b-a)+(a \vee b-b)=|b-a|$.

For all $n \in \mathbb{N}$, we have

$$
\begin{aligned}
b=\alpha \otimes^{\text {oag }}(1 / \alpha) & \leq p_{2 n+1} / q_{2 n+1} \otimes^{\text {oag }} q_{2 n} / p_{2 n} \\
& =\left(p_{2 n} / q_{2 n}+\rho_{n}\right) \otimes^{\text {oag }} q_{2 n} / p_{2 n} \\
& =\left(p_{2 n} / q_{2 n}\right) \otimes^{\text {oag }}\left(q_{2 n} / p_{2 n}\right)+\rho_{n} \otimes^{\text {oag }}\left(q_{2 n} / p_{2 n}\right) \\
& =a+\rho_{n} \otimes^{\text {oag }}\left(q_{2 n} / p_{2 n}\right) \quad(\text { by Claim } 2),
\end{aligned}
$$

thus $n(b-a) \leq\left(n \rho_{n}\right) \otimes^{\text {oag }}\left(q_{2 n} / p_{2 n}\right) \leq 1 \otimes^{\text {oag }} 2=2 a$. Similarly, one proves that $-2 a \leq n(b-a)$ : thus we have obtained that

$$
(\forall n \in \mathbb{N})(-2 a \leq n(b-a) \leq 2 a) .
$$

Therefore, we also have

$$
(\forall n \in \mathbb{N})(0 \leq n c \leq 2 a)
$$

(recall that $c=|b-a|$ ). However, there exists a unique positive homomorphism $\phi$ from $\mathbb{R} \otimes^{\text {oag }} \mathbb{R}$ to $\mathbb{R}$ such that $(\forall x, y \in \mathbb{R})\left(\phi\left(x \otimes^{\text {oag }} y\right)=x y\right)$, and $(* *)$ would imply that for all $n \in \mathbb{N}, 0 \leq n \phi(|b-a|) \leq 2$, whence $\phi(|b-a|)=0$. But by definition of the positive cone of $\mathbb{R} \otimes^{\text {oag }} \mathbb{R}$, it is easy to see that $\operatorname{ker}(\phi) \cap\left(\mathbb{R} \otimes^{\text {oag }} \mathbb{R}\right)^{+}=\{0\}$, whence $|b-a|=0$, i.e. $a=b$. However, if $a=b$, i.e. (see Proposition 1.1) $1 \otimes^{\mathrm{ag}} 1=\alpha \otimes^{\mathrm{ag}}(1 / \alpha)$, then, by Claim 1 , there exist $p, q \in \mathbb{Z} \backslash\{0\}$ such that $p=q \alpha$, a contradiction since $\alpha$ is irrational.

Note that the proof above yields in fact that if $\alpha$ is an irrational number and $A$ and $B$ are two additive subgroups of $\mathbb{R}$ such that $\{1, \alpha\} \subseteq A$ and $\{1,1 / \alpha\} \subseteq B$, then $A \otimes^{\text {oag }} B$ is not lattice-ordered. A similar proof yields also the same negative result for the symmetrical power of $\mathbb{R}$ of order 2 . However, the same technique as it is used in the proof of [5, Proposition 2.3] yields the following result: 
Proposition 1.7. Let $A$ be an unperforated interpolation group (not necessarily directed) and let $B$ be an interpolation group. Then $A \otimes^{\text {oag }} B$ is an interpolation group.

Proof. Since the satisfaction by a partially ordered abelian group of the interpolation property depends only on its positive cone, it results from Proposition 1.2, (b, iii) that it suffices to consider the case where both $A$ and $B$ are directed. But then, $A$ is a dimension group, thus, by the theorem of Effros, Handelman and Shen ([2, Theorem 2.2], or [6, Theorem 3.19]), it is a direct limit of simplicial groups (i.e. partially ordered abelian groups isomorphic to some $\mathbb{Z}^{n}$ with its natural ordering); since $\otimes^{\text {oag }}$ commutes with the direct limit operation [6, Lemma 2.2], it suffices to prove the theorem for $A=\mathbb{Z}^{n}, n \in \mathbb{Z}^{+}$. But in this case, $A \otimes^{\text {oag }} B \cong B^{n}$ and the conclusion follows.

Problem 1.8. For every perforated interpolation group $A$, prove that there exists a directed torsion-free interpolation group $B$ such that $A \otimes^{\text {oag }} B$ does not have interpolation.

\section{Case of commutative monoids.}

In this paragraph we shall prove that the finite refinement property is preserved under tensor products of commutative monoids. The essential part of the proof aims at finding wieldy enough necessary and sufficient conditions for an equality $\sum_{i<m} a_{i} \otimes^{\mathrm{cm}} b_{i}=\sum_{j<n} a_{j}^{\prime} \otimes^{\mathrm{cm}} b_{j}^{\prime}$ to hold in a tensor product of refinement monoids. Although such conditions have been announced by P.A. Grillet in [8, Theorem 4] (in the context of commutative semigroups), we could not use these for our problem, thus we shall introduce a different, more symmetric criterion of equality of two tensors.

We start first with conical refinement monoids. For every conical commutative monoid $A$, put $A^{\bullet}=A \backslash\{0\}$. By definition of conicality, $A^{\bullet}$ is a subsemigroup of $A$.

Now, if $A$ is an arbitrary set and $R$ is a binary relation on $A$, then $A$ is

- confluent when for all $a, b, b^{\prime}$ in $A$ such that $a R b$ and $a R b^{\prime}$, there exists $c \in A$ such that $b R c$ and $b^{\prime} R c$.

If in addition $A$ is a commutative monoid, say that $R$ is

- additive when for all $a, a^{\prime}, b, b^{\prime}$ in $A,\left(a R a^{\prime}\right.$ and $\left.b R b^{\prime}\right)$ implies $a+b R a^{\prime}+b^{\prime}$

- refining when for all $a, b, c$ in $A$ such that $a+b R c$, there are $a^{\prime}$ and $b^{\prime}$ in $A$ such that $a R a^{\prime}$ and $b R b^{\prime}$ and $c=a^{\prime}+b^{\prime}$. 
Now let $A$ and $B$ be two conical refinement monoids, which we shall fix from 2.1 to 2.6. Let $\mathcal{C}=\mathbb{Z}^{\left(A^{\bullet} \times B^{\bullet}\right)}$ be the abelian group of all functions $f: A^{\bullet} \times B^{\bullet} \rightarrow \mathbb{Z}$ such that $f^{-1}\{0\}$ is cofinite, ordered componentwise. Thus $\mathcal{C}$ is a [Dedekind complete] lattice-ordered group. For all $(a, b) \in A^{\bullet} \times B^{\bullet}$, let $a \cdot b$ be the element of $\mathcal{C}^{+}$defined by $(a \cdot b)((x, y))=1$ if $(x, y)=(a, b), 0$ otherwise. Thus the elements of $\mathcal{C}^{+}$are exactly the finite sums of elements of the form $a \cdot b$ where $(a, b) \in A^{\bullet} \times B^{\bullet}\left(\mathcal{C}^{+}\right.$is the free commutative monoid on $\left.A^{\bullet} \times B^{\bullet}\right)$. Define binary relations $\rightarrow^{0}, \rightarrow$ and $\rightarrow^{*}$ on $\mathcal{C}^{+}$the following way:

- $f \rightarrow^{0} g$ if and only if there are $(a, b) \in A^{\bullet} \times B^{\bullet}$, nonempty finite sets $I$ and $J$ and $\left(a_{i}, b_{j}\right) \in A^{\bullet} \times B^{\bullet}($ all $(i, j) \in I \times J)$ such that $a=\sum_{i \in I} a_{i}$, $b=\sum_{j \in J} b_{j}$ and $f=a \cdot b$ and $g=\sum_{(i, j) \in I \times J} a_{i} \cdot b_{j}$;

- $f \rightarrow g$ if and only if there are $n \in \mathbb{Z}^{+}, f_{i}, g_{i}(i<n)$ in $\mathcal{C}^{+}$such that $f=\sum_{i<n} f_{i}, g=\sum_{i<n} g_{i}$ and $f_{i} \rightarrow^{0} g_{i}$ for all $i<n$,

- $f \rightarrow^{*} g$ if and only if there are $n \in \mathbb{N}$ and $h_{i}(i \leq n)$ in $\mathcal{C}^{+}$such that $h_{0}=f, h_{n}=g$ and for all $i<n, h_{i} \rightarrow h_{i+1}$ (that is, $\rightarrow^{*}$ is the transitive closure of $\rightarrow$ ).

Note that in the definition of $\rightarrow$, the $f_{i}$ 's are necessarily of the form $a_{i} \cdot b_{i}$, thus the expression of $f$ as $\sum_{i<n} f_{i}$ is essentially unique. This implies easily (iv) of the following lemma (the rest is trivial):

Lemma 2.1. The following holds:

(i) For all $(a, b) \in A^{\bullet} \times B^{\bullet}$, we have $a \cdot b \rightarrow^{0} a \cdot b$.

(ii) The relation $\rightarrow$ contains $\rightarrow^{0}$ and it is reflexive.

(iii) The relation $\rightarrow$ is additive.

(iv) The relation $\rightarrow$ is refining.

The following lemma follows easily:

Lemma 2.2. The relation $\rightarrow^{*}$ is reflexive, transitive, additive and refining.

Now we shall prove the main lemma of this section:

Lemma 2.3. Both relations $\rightarrow$ and $\rightarrow^{*}$ are confluent.

Proof. Since $\rightarrow^{*}$ is the transitive closure of $\rightarrow$, it suffices to prove that $\rightarrow$ is confluent (in the language of the theory of rewriting rules, this is usually expressed as "local confluency implies confluency"). Thus let $f, g, g^{\prime}$ in $\mathcal{C}^{+}$such that $f \rightarrow g$ and $f \rightarrow g^{\prime}$, we prove that there exists $h \in \mathcal{C}^{+}$such that $g \rightarrow h$ and $g^{\prime} \rightarrow h$. Let us first see the case where $f=a \cdot b$ where $(a, b) \in A^{\bullet} \times B^{\bullet}$. Thus $f \rightarrow^{0} g$ and $f \rightarrow^{0} g^{\prime}$, thus there are nonempty finite 
sets $I, I^{\prime}, J, J^{\prime}$ such that $I \cap I^{\prime}=J \cap J^{\prime}=\emptyset$ and elements $a_{k}\left(k \in I \cup I^{\prime}\right)$ and $b_{l}\left(l \in J \cup J^{\prime}\right)$ such that $a=\sum_{i \in I} a_{i}=\sum_{i^{\prime} \in I^{\prime}} a_{i^{\prime}}, b=\sum_{j \in J} b_{j}=\sum_{j^{\prime} \in J^{\prime}} b_{j^{\prime}}$ and $g=\sum_{(i, j) \in I \times J} a_{i} \cdot b_{j}$ and $g^{\prime}=\sum_{\left(i^{\prime}, j^{\prime}\right) \in I^{\prime} \times J^{\prime}} a_{i^{\prime}} \cdot b_{j^{\prime}}$. Since both $A$ and $B$ satisfy the finite refinement property, there are elements $a_{i i^{\prime}}\left(\left(i, i^{\prime}\right) \in I \times I^{\prime}\right)$ of $A$ and $b_{j j^{\prime}}\left(\left(j, j^{\prime}\right) \in J \times J^{\prime}\right)$ such that the following holds:

$$
(\forall i \in I)\left(a_{i}=\sum_{i^{\prime} \in I^{\prime}} a_{i i^{\prime}}\right) \quad \text { and }\left(\forall i^{\prime} \in I^{\prime}\right)\left(a_{i^{\prime}}=\sum_{i \in I} a_{i i^{\prime}}\right)
$$

and

$$
(\forall j \in J)\left(b_{j}=\sum_{j^{\prime} \in J^{\prime}} b_{j j^{\prime}}\right) \quad \text { and }\left(\forall j^{\prime} \in J^{\prime}\right)\left(b_{j^{\prime}}=\sum_{j \in J} b_{j j^{\prime}}\right) .
$$

Note that some of the $a_{i i^{\prime}}$ 's or the $b_{j j^{\prime}}$ 's may be zero. Thus for all $i \in I$ (resp. $i^{\prime} \in I^{\prime}, j \in J, j^{\prime} \in J^{\prime}$ ), define respectively

$$
\begin{aligned}
& I_{i}^{\prime}=\left\{i^{\prime} \in I^{\prime}: a_{i i^{\prime}} \neq 0_{A}\right\} \quad \text { and } \quad I_{i^{\prime}}=\left\{i \in I: a_{i i^{\prime}} \neq 0_{A}\right\} \text {, } \\
& J_{j}^{\prime}=\left\{j^{\prime} \in J^{\prime}: b_{j j^{\prime}} \neq 0_{B}\right\} \quad \text { and } \quad J_{j^{\prime}}=\left\{j \in J: b_{j j^{\prime}} \neq 0_{B}\right\} \text {. }
\end{aligned}
$$

Now we prove a

Claim. For all $(i, j) \in I \times J\left(\right.$ resp. $\left.\left(i^{\prime}, j^{\prime}\right) \in I^{\prime} \times J^{\prime}\right)$, we have $a_{i} \cdot b_{j} \rightarrow^{0}$ $\sum_{\left(i^{\prime}, j^{\prime}\right) \in I_{i}^{\prime} \times J_{j}^{\prime}} a_{i i^{\prime}} \cdot b_{j j^{\prime}}\left(\right.$ resp. $\left.a_{i^{\prime}} \cdot b_{j^{\prime}} \rightarrow^{0} \sum_{(i, j) \in I_{i^{\prime}} \times J_{j^{\prime}}} a_{i i^{\prime}} \cdot b_{j j^{\prime}}\right)$.

Proof. We prove for example the first assertion. Thus let $(i, j) \in I \times J$. Then we have $a_{i}=\sum_{i^{\prime} \in I^{\prime}} a_{i i^{\prime}}=\sum_{i^{\prime} \in I_{i}^{\prime}} \underbrace{a_{i i^{\prime}}}_{\neq 0_{A}}$ and similarly, $b_{j}=\sum_{j^{\prime} \in J_{j}^{\prime}} \underbrace{b_{j j^{\prime}}}_{\neq 0_{B}}$ Therefore, by definition of $\rightarrow^{0}, a_{i} \cdot b_{j} \rightarrow^{0} \sum_{\left(i^{\prime}, j^{\prime}\right) \in I_{i}^{\prime} \times J_{j}^{\prime}} a_{i i^{\prime}} \cdot b_{j j^{\prime}}$.

Now put $I^{*}=\left\{\left(i, i^{\prime}\right) \in I \times I^{\prime}: a_{i i^{\prime}} \neq 0_{A}\right\}$ and $J^{*}=\left\{\left(j, j^{\prime}\right) \in J \times J^{\prime}:\right.$ $\left.b_{j j^{\prime}} \neq 0_{B}\right\}$. Put $h=\sum_{\left(\left(i, i^{\prime}\right),\left(j, j^{\prime}\right)\right) \in I^{*} \times J^{*}} a_{i i^{\prime}} \cdot b_{j j^{\prime}}$. By the claim above and by definition of $\rightarrow$, we have

$$
g=\sum_{(i, j) \in I \times J} a_{i} \cdot b_{j} \rightarrow \sum_{(i, j) \in I \times J} \sum_{\left(i^{\prime}, j^{\prime}\right) \in I_{i}^{\prime} \times J_{j}^{\prime}} a_{i i^{\prime}} \cdot b_{j j^{\prime}}=\sum_{\left(\left(i, i^{\prime}\right),\left(j, j^{\prime}\right)\right) \in I^{*} \times J^{*}} a_{i i^{\prime}} \cdot b_{j j^{\prime}}=h .
$$

Similarly, $g^{\prime} \rightarrow h$. Thus in this particular case, we have confluency. In the general case, one can write $f=\sum_{i<n} f_{i}, g=\sum_{i<n} g_{i}$ and $g^{\prime}=\sum_{i<n} g_{i}^{\prime}$ where $n \in \mathbb{Z}^{+}$, the $f_{i}$ 's are of the form $a_{i} \cdot b_{i}\left(\left(a_{i}, b_{i}\right) \in A^{\bullet} \times B^{\bullet}\right), g_{i}, g_{i}^{\prime} \in \mathcal{C}^{+}$ (all $i<n$ ) and $f_{i} \rightarrow^{0} g_{i}$ and $f_{i} \rightarrow^{0} g_{i}^{\prime}$ for all $i<n$. By the previous study, 
there are $h_{i}(i<n)$ in $\mathcal{C}^{+}$such that for all $i<n, g_{i} \rightarrow h_{i}$ and $g_{i}^{\prime} \rightarrow h_{i}$. Put $h=\sum_{i<n} h_{i}$. Then $g \rightarrow h$ and $g^{\prime} \rightarrow h$.

Now we define a binary relation $\equiv$ on $\mathcal{C}^{+}$the following way:

$$
f \equiv g \Longleftrightarrow\left(\exists h \in \mathcal{C}^{+}\right)\left(f \rightarrow^{*} h \text { and } g \rightarrow^{*} h\right) .
$$

Since $\rightarrow^{*}$ is reflexive, it is immediate that $\equiv$ is reflexive and symmetric. Since $\rightarrow^{*}$ is transitive and, by previous lemma, confluent, $\equiv$ is transitive. Since $\rightarrow^{*}$ is additive, we obtain the following

Lemma 2.4. The binary relation $\equiv$ is a congruence on $\mathcal{C}^{+}$.

Now, let $C$ be the quotient monoid of $\mathcal{C}^{+}$under $\equiv$. For all $f \in \mathcal{C}^{+}$, denote by $[f]$ the equivalence class of $f$ under $\equiv$. For all $(a, b) \in A \times B$, define an element $a \odot b$ of $C$ the following way:

$$
a \odot b= \begin{cases}{[a \cdot b]} & \text { if } a \neq 0_{A} \text { and } b \neq 0_{B}, \\ 0_{C} & \text { otherwise. }\end{cases}
$$

Lemma 2.5. The monoid $C$ is a conical refinement monoid, and for all $(a, b) \in A^{\bullet} \times B^{\bullet}$, we have $a \odot b \neq 0_{C}$.

Proof. Note that for all $f \in \mathcal{C}^{+}, 0 \rightarrow f$ implies $f=0$; thus $[0]=\{0\}$, so that if $n \in \mathbb{N}$ and $\left(a_{i}, b_{i}\right)(i<n)$ are elements of $A^{\bullet} \times B^{\bullet}$, then $\sum_{i<n} a_{i} \cdot b_{i} \not \equiv 0$. The conicality statement as well as the last statement follow. It remains to prove that $C$ satisfies the finite refinement property.

Thus let $f_{0}, f_{1}, g_{0}, g_{1}$ in $\mathcal{C}^{+}$such that $\left[f_{0}\right]+\left[f_{1}\right]=\left[g_{0}\right]+\left[g_{1}\right]$; by definition, $f_{0}+f_{1} \equiv g_{0}+g_{1}$, i.e. there exists $h \in \mathcal{C}^{+}$such that $f_{0}+f_{1} \rightarrow^{*} h$ and $g_{0}+g_{1} \rightarrow^{*} h$. Since $\rightarrow^{*}$ is refining (Lemma 2.2), there are $f_{i}^{\prime}, g_{i}^{\prime}(i<2)$ in $\mathcal{C}^{+}$ such that for all $i<2, f_{i} \rightarrow^{*} f_{i}^{\prime}$ and $g_{i} \rightarrow^{*} g_{i}^{\prime}$ and $h=f_{0}^{\prime}+f_{1}^{\prime}=g_{0}^{\prime}+g_{1}^{\prime}$. But since $\mathcal{C}$ is a lattice-ordered group, $\mathcal{C}^{+}$satisfies the finite refinement property, thus there are $h_{i j}(i, j<2)$ in $\mathcal{C}^{+}$such that for all $i<2$, one has $f_{i}^{\prime}=h_{i 0}+h_{i 1}$ and $g_{i}^{\prime}=h_{0 i}+h_{1 i}$. It follows that for all $i<2$, we also have $\left[f_{i}\right]=\left[h_{i 0}\right]+\left[h_{i 1}\right]$ and $\left[g_{i}\right]=\left[h_{0 i}\right]+\left[h_{1 i}\right]$, which completes the proof.

Now, the proof for conical refinement monoids will be completed by the following

Lemma 2.6. The map $\odot$ is a universal bimorphism.

We first prove that $\odot$ is a bimorphism. Thus let us prove for example that for all $a_{0}, a_{1} \in A$ and all $b \in B$, we have $\left(a_{0}+a_{1}\right) \odot b=a_{0} \odot b+a_{1} \odot b$. It 
is trivial when $a_{0}=0_{A}$ or $a_{1}=0_{A}$ or $b=0_{B}$; otherwise, since $A$ is conical, $a_{0}+a_{1} \neq 0_{A}$, and $\left(a_{0}+a_{1}\right) \cdot b \rightarrow a_{0} \cdot b+a_{1} \cdot b ;$ whence $\left[\left(a_{0}+a_{1}\right) \cdot b\right]=\left[a_{0} \cdot b\right]+\left[a_{1} \cdot b\right]$. The proof is similar for the roles of $A$ and $B$ exchanged.

We now verify that $\odot$ is universal. Thus let $D$ be a commutative monoid and let $\phi: A \times B \rightarrow D$ be a bimorphism. Define a map $\psi$ from $\mathcal{C}^{+}$to $D$ by the rule

$$
\psi\left(\sum_{i<n} a_{i} \cdot b_{i}\right)=\sum_{i<n} \phi\left(a_{i}, b_{i}\right) .
$$

Thus $\psi$ is a monoid homomorphism from $\mathcal{C}^{+}$to $D$.

Claim. For all $f, g \in \mathcal{C}^{+}, f \rightarrow^{*} g$ implies that $\psi(f)=\psi(g)$.

Proof. By definition of $\rightarrow^{*}$, it suffices to prove that $f \rightarrow g$ implies $\psi(f)=$ $\psi(g)$. Since $\psi$ is a monoid homomorphism, it suffices to prove that $f \rightarrow^{0} g$ implies $\psi(f)=\psi(g)$. Thus write $f=a \cdot b, a=\sum_{i \in I} a_{i}, b=\sum_{j \in J} b_{\jmath}$, where $I, J$ are nonempty finite sets and $(a, b)$ and the $\left(a_{i}, b_{j}\right)$ 's are elements of $A^{\bullet} \times B^{\bullet}$, with $g=\sum_{(i, j) \in I \times J} a_{i} \cdot b_{j}$. Therefore, we have

$$
\begin{aligned}
\psi(g) & =\sum_{(i, j) \in I \times J} \phi\left(a_{i}, b_{j}\right) \\
& =\phi\left(\sum_{i \in I} a_{i}, \sum_{j \in J} b_{j}\right) \\
& =\phi(a, b) \\
& =\psi(f) .
\end{aligned}
$$

It follows from the claim that $\psi$ is constant on all $\equiv$-equivalence classes; therefore, there exists a monoid homomorphism $\bar{\phi}: C \rightarrow D$ such that $(\forall f \in$ $\left.\mathcal{C}^{+}\right)(\bar{\phi}([f])=\psi(f))$. In particular, for all $(a, b) \in A^{\bullet} \times B^{\bullet}, \bar{\phi}(a \odot b)=$ $\psi(a \cdot b)=\phi(a, b)$; for $a=0_{A}$ or $b=0_{B}$, we have $\bar{\phi}(a \odot b)=\phi(a, b)=0$, whence $\bar{\phi}(a \odot b)=\phi(a, b)$ for all $(a, b) \in A \times B$. Uniqueness of $\bar{\phi}$ follows from the fact that $C$ is generated (as a monoid) by $\left\{a \odot b:(a, b) \in A^{\bullet} \times B^{\bullet}\right\}$.

Now, Lemmas 2.5 and 2.6 allow us to identify $A \otimes \otimes^{\mathrm{cm}} B$ with $C$, and to write $a \otimes^{\mathrm{cm}} b$ instead of $a \odot b$. Thus we state the following theorem:

Theorem 2.7. Let $A$ and $B$ be two conical refinement monoids. Then $A \otimes^{\mathrm{cm}} B$ is a conical refinement monoid, and for all $(a, b) \in A^{\bullet} \times B^{\bullet}$, we have $a \otimes^{\mathrm{cm}} b \neq 0$.

It remains to extend Theorem 2.7 to refinement monoids that are non necessarily conical. We first need a 
Lemma 2.8. Let $M$ be a commutative monoid and let $N$ be a submonoid of $M$. Define a binary relation $\approx_{N}$ on $M$ by putting

$$
x \approx_{N} y \Leftrightarrow(\exists u, v \in N)(x+u=y+v) .
$$

Then $\approx_{N}$ is a congruence on $M$. Denote by $M / N$ the quotient monoid of $M$ under $\approx_{N}$. If $M$ is a refinement monoid, then $M / N$ is a refinement monoid.

Proof. The fact that $\approx_{N}$ is a congruence on $M$ is trivial. For all $x \in M$, denote by $x / N$ the equivalence class of $x$ modulo $\approx_{N}$. Let $x_{0}, x_{1}, y_{0}, y_{1}$ in $M$ such that $x_{0} / N+x_{1} / N=y_{0} / N+y_{1} / N$. By definition, there are $u, v \in N$ such that $x_{0}+x_{1}+u=y_{0}+y_{1}+v$. Since $M$ is a refinement monoid, there are $z_{i j}(i, j<2)$ such that $x_{0}=z_{00}+z_{01}, x_{1}+u=z_{10}+z_{11}, y_{0}=z_{00}+z_{10}$ and $y_{1}+v=z_{01}+z_{11}$. Since $x_{1} / N=\left(x_{1}+u\right) / N$ and $y_{1} / N=y_{1}+v / N$, we also have $x_{0} / N=z_{00} / N+z_{01} / N, x_{1} / N=z_{10} / N+z_{11} / N, y_{0} / N=z_{00} / N+z_{10} / N$ and $y_{1} / N=z_{01} / N+z_{11} / N$. Thus $M / N$ is a refinement monoid.

Theorem 2.9. Let $A$ and $B$ be refinement monoids. Then $A \otimes^{\mathrm{cm}} B$ is a refinement monoid.

Proof. For every semigroup $S$, let $S^{0}$ be the monoid obtained by adjoining a new unit element 0 to $S$ (thus, in the case where $S$ has already a unit $0_{S}$, we have $0_{S} \neq 0$ ). Observe that a commutative monoid is conical if and only if it is of the form $S^{0}$ for a certain commutative semigroup $S$. Now, if $A$ and $B$ are refinement monoids, it is obvious that both $A^{0}$ and $B^{0}$ are conical refinement monoids. By Theorem 2.7, $M=A^{0} \otimes^{\mathrm{cm}} B^{0}$ is a conical refinement monoid. Denote by $(a, b) \mapsto a \otimes^{0} b$ the natural map from $A^{0} \times B^{0}$ to $M$. Define a submonoid $N$ of $M$ by putting

$$
N=\{0\} \cup\left\{a \otimes^{0} 0_{B}+0_{A} \otimes^{0} b:(a, b) \in A \times B\right\} .
$$

Now define a map $\odot$ from $A \times B$ to $M / N$ by putting $a \odot b=a \otimes^{0} b / N$. Using the definition of $N$, it is easily seen that $\odot$ is a bimorphism. Let us now prove that $\odot$ is universal.

Thus let $D$ be a commutative monoid and let $f: A \times B \rightarrow D$ be a bimorphism. Then the natural extension $f^{0}$ of $f$ from $A^{0} \times B^{0}$ to $D$ defined by the rule

$$
f^{0}(a, b)= \begin{cases}f(a, b) & \text { if }(a, b) \in A \times B, \\ 0_{D} & \text { otherwise }\end{cases}
$$

is easily seen to be a bimorphism from $A^{0} \times B^{0}$ to $D$. Thus there exists a unique monoid homomorphism $g^{0}$ from $M$ to $D$ such that $(\forall(a, b) \in$ $\left.A^{0} \times B^{0}\right)\left(g^{0}\left(a \otimes^{0} b\right)=f^{0}(a, b)\right)$. But by definition of $f^{0}$ and of $N$, we 
have $g^{0} \uparrow_{N}=0$, whence $g^{0}$ is constant on the equivalence classes of $\approx_{N}$. Thus there exists a unique monoid homomorphism $g: M / N \rightarrow D$ such that $(\forall x \in M)\left(g(x / N)=g^{0}(x)\right)$. It follows that for all $(a, b) \in A \times B$, we have $g(a \odot b)=g^{0}\left(a \otimes^{0} b\right)=f^{0}(a, b)=f(a, b)$. Since $M / N$ is generated by the $a \odot b$ 's, uniqueness of $g$ follows. Thus we have proved the universality of $\odot$.

Hence, $A \otimes^{\mathrm{cm}} B$ is isomorphic to $M / N$, so that, by Lemma 2.8 , it is a refinement monoid.

We shall now present a small application of the definition of $\equiv$ through the confluent relation $\rightarrow$. For every commutative monoid $A$, define a binary relation $\asymp$ on $A$ by putting

$$
x \asymp y \Longleftrightarrow(\exists n \in \mathbb{N})\left(x \leq_{\text {alg }} n y \text { and } y \leq_{\text {alg }} n x\right) .
$$

It is easy to verify that $\asymp$ is a congruence on $A$ satisfying $(\forall x)(x \asymp 2 x)$. Therefore, the quotient monoid $\hat{A}=A / \asymp$ is a semilattice (i.e. a commutative monoid satisfying $(\forall x)(x=2 x))$; one can in fact easily verify that $\hat{A}$ is the universal semilattice of $A$. Denote by $a \mapsto \hat{a}^{A}$ the natural homomorphism from $A$ onto $\hat{A}$.

Proposition 2.10. Let $A$ and $B$ be conical refinement monoids. Then there are unique monoid homomorphisms $p: A \otimes^{\mathrm{cm}} B \rightarrow \hat{A}$ and $q: A \otimes^{\mathrm{cm}} B \rightarrow \hat{B}$ (the projections) such that for all $(a, b)$ in $A^{\bullet} \times B^{\bullet}$, we have $p\left(a \otimes^{\mathrm{cm}} b\right)=\hat{a}^{A}$ and $q\left(a \otimes^{\mathrm{cm}} b\right)=\hat{b}^{B}$.

Proof. If $A=\left\{0_{A}\right\}$ or $B=\left\{0_{B}\right\}$ then it is vacuously true, thus suppose that both $A$ and $B$ are non zero. Let us use the notations as in the proof of Theorem 2.7. Let us prove for example the assertion for $p$. For all $f=$ $\sum_{i<n} a_{i} \cdot b_{i}\left(n \in \mathbb{Z}^{+},\left(a_{i}, b_{i}\right) \in A^{\bullet} \times B^{\bullet}\right)$, put $L(f)=\sum_{i<n} a_{i}$. Then it clearly suffices to prove that for all $f, g \in \mathcal{C}^{+}, f \equiv g$ implies $L(f) \asymp L(g)$. By definition of $\equiv$, it suffices to prove this for $f \rightarrow g$, thus for $f \rightarrow^{0} g$. Thus put $f=a \cdot b, g=\sum_{(i, j) \in I \times J} a_{i} \cdot b_{j}$ with $I$ and $J$ finite nonempty, $(a, b)$ and the $\left(a_{i}, b_{j}\right)$ 's in $A^{\bullet} \times B^{\bullet}$ with $a=\sum_{i \in I} a_{i}$ and $b=\sum_{j \in J} b_{j}$. Thus we have $L(g)=\sum_{i \in I}|J| a_{i}=|J| \sum_{i \in I} a_{i}=|J| a=|J| L(f) \asymp L(f)$ and the conclusion follows.

Corollary 2.11. Let $A$ and $B$ be conical refinement monoids and let $a_{0}, a_{1} \in A$ and $b \in B$. If $a_{0} \perp_{A} a_{1}$, then $a_{0} \otimes^{\mathrm{cm}} b \perp_{A \otimes^{\mathrm{cm} B}} a_{1} \otimes^{\mathrm{cm}} b$.

Proof. If $a_{0}=0_{A}$ or $a_{1}=0_{A}$ or $b=0_{B}$ then there is nothing to prove, so suppose that $a_{0}, a_{1}$ and $b$ are non zero. Let $p: A \otimes^{\mathrm{cm}} B \rightarrow \hat{A}$ be the first projection as defined in previous proposition. Let $c \in A \otimes^{\mathrm{cm}} B$ such that $c \leq a_{0} \otimes^{\mathrm{cm}} b, a_{1} \otimes^{\mathrm{cm}} b$. Then, in $\hat{A}$, we have $p(c) \leq_{\text {alg }} p\left(a_{i} \otimes^{\mathrm{cm}} b\right)=\hat{a}_{i}^{A}$ 
for all $i<2$, thus there exist $n \in \mathbb{N}$ and $d \in p(c)$ such that $A$ satisfies $d \leq_{\text {alg }} n a_{0}, n a_{1}$. Since $a_{0} \perp_{A} a_{1}$ and $A$ is a refinement monoid, it follows that $d=0_{A}$, thus $p(c)=0_{\hat{A}}$; but this is possible only when $c=0_{A \otimes^{\mathrm{cm} B}}$.

As the proof of Example 1.5 shows, it is not possible to generalize this to the tensor product of interpolation groups (for $b \geq 0$ ), even in the case where both groups are directed and torsion-free: indeed, $A \otimes^{\text {oag }} B$ satisfies $0,-a \otimes^{\text {oag }} \varepsilon \leq a_{0} \otimes^{\text {oag }}(1+\varepsilon), a_{1} \otimes^{\text {oag }}(1+\varepsilon)$ and $a_{0} \perp_{A} a_{1}$, but $-a \otimes^{\text {oag }} \varepsilon \not \leq 0$ (otherwise there would be a trivial interpolant - namely $0 .$. ). On the other hand, in the unperforated case, we still can conclude by using the result on monoids:

Corollary 2.12. Let $A$ and $B$ be two interpolation groups one of which is unperforated, let $a_{0}, a_{1} \in A^{+}$and let $b \in B^{+}$such that $a_{0} \perp_{A} a_{1}$. Then $a_{0} \otimes^{\text {oag }} b \perp_{A \otimes^{\text {oag } B}} a_{1} \otimes^{\text {oag }} b$.

Proof. By Proposition 1.2, (b, iii), it suffices to consider the case where both $A$ and $B$ are directed; in that case, by Proposition 1.2 (a), it suffices to prove that $A^{+} \otimes^{\mathrm{cm}} B^{+}$is cancellative (because then, $\left(A \otimes^{\text {oag }} B\right)^{+} \cong A^{+} \otimes^{\mathrm{cm}} B^{+}$and we can apply the result on monoids). But by a straightforward application of the Effros, Handelman and Shen theorem, every positive cone of a dimension group is a direct limit (as a monoid) of positive cones of simplicial groups (say simplicial cones); thus if for example $A$ is a dimension group, then $A^{+}$is a direct limit of simplicial cones, and since $\otimes^{\text {oag }}$ commutes with the operation of direct limit, it suffices to conclude when $A^{+}$is a simplicial cone. But if $A=\left(\mathbb{Z}^{+}\right)^{n}$, then $A^{+} \otimes^{\mathrm{cm}} B^{+} \cong\left(B^{+}\right)^{n}$, and this monoid is cancellative; and similarly with the roles of $A$ and $B$ exchanged.

Remark 2.13. In particular, in Examples 1.4 and 1.5 are obtained directed interpolation groups $A$ and $B$ such that $A \otimes^{\text {oag }} B$ does not have interpolation. But by Theorem 2.7, $A^{+} \otimes^{\mathrm{cm}} B^{+}$satisfies the finite refinement property; thus, by Proposition 1.1 (a), it is a refinement monoid whose Grothendieck group is not an interpolation group. Such a situation has already been encountered in [10]: if $R$ is a regular ring, then $K_{0}(R)$ is the Grothendieck group of the monoid $V(R)$ of all isomorphism types of finitely generated projective right $R$-modules; although $V(R)$ is always a refinement monoid, [10] shows an example where its Grothendieck group is not an interpolation group.

On the other hand, there are directed partially ordered abelian groups which cannot be [isomorphic to] the Grothendieck group of any refinement monoid: in particular, when there is an order-unit, then the latter is always an asymptotic interpolation group as defined in [11]. In particular, the tensor product (in the category of partially ordered abelian groups) of finitely many interpolation groups with order-unit is an asymptotic interpolation group. 
Problem 2.14. Say that a directed partially ordered abelian group $G$ is ratio$n a l$ when it is torsion-free and satisfies $\left(\forall x, y \in G^{+} \backslash\{0\}\right)(\exists m, n \in \mathbb{N})(m x=$ $n y)$. Thus rational partially ordered abelian groups are exactly those directed partially ordered abelian groups whose positive cone is a submonoid of $\mathbb{Q}^{+}$. Note that the tensor product $\left(\otimes^{\text {oag }}\right)$ of two rational partially ordered abelian groups is a rational partially ordered abelian group. One can easily show that the partially ordered abelian groups constructed by E. Pardo in [12] are rational (yielding there an example of rational partially ordered abelian group which is in addition a strictly perforated, torsion-free, simple Riesz group). This is also mentioned without proof in [13, Example 1]. Now our problem is: find a rational partially ordered abelian group $G$ with interpolation such that $G \otimes^{\text {oag }} G$ does not satisfy interpolation. Is there a general way to decide this kind of statement? Is there a "calculus" of rational partially ordered abelian groups?

Problem 2.15. Are rational interpolation groups in some sense "building blocks" (through some kind of "limit"?) of all simple torsion-free interpolation groups?

Further note. Ken Goodearl communicated us a simplification of Example 1.5, bringing Examples 1.3 to 1.5 into a natural sequence.

\section{References}

[1] A. Bigard, K. Keimel and S. Wolfenstein, Groupes et anneaux réticulés, Lecture Notes in Mathematics, Springer-Verlag, 608 (1977).

[2] E.G. Effros, D.E. Handelman and C.-L. Shen, Dimension groups and their affine representations, American Journal of Mathematics, 102(2) (1980), 385-407.

[3] D.H. Fremlin, Tensor products of Archimedean vector lattices, American Journal of Mathematics, 94 (1972), 777-798.

[4] L. Fuchs, Infinite abelian groups, Academic Press, I (1970) and II (1973).

[5] K.R. Goodearl and D.E. Handelman, Tensor products of dimension groups and $K_{0}$ of unit-regular rings, Canadian Journal of Mathematics, 38(3) (1986), 633-658.

[6] K.R. Goodearl, Partially ordered abelian groups with the interpolation property, Mathematical surveys and monographs, 20, American Mathematical Society, 1986.

[7] P.A. Grillet, The tensor product of commutative semigroups, Transactions of the American Mathematical Society, 138 (1969), 281-293.

[8] - Interpolation properties and tensor products of semigroups, Semigroup Forum, 1 (1970), 162-168.

[9] J. Martinez, Tensor products of partially ordered abelian groups, Pacific Journal of Mathematics, 41 (1972), 771-789.

[10] J. Moncasi, A regular ring whose $K_{0}$ is not a Riesz group, Communications in Algebra, 13 (1985), 125-133. 
[11] E. Pardo, Metric completions of ordered groups and $K_{0}$-theory of exchange rings, to appear in Trans. Amer. Math. Soc.

[12] - On the classification of simple Riesz groups, preprint.

[13] F. Wehrung, Metric properties of positively ordered monoids, Forum Mathematicum, 5 (1993), 183-201.

Received October 13, 1994 and revised May 10, 1995.

University de CAen

14032 Caen Cedex

FrANCE

E-mail address: gremlin@math.unicaen.fr 



\title{
PACIFIC JOURNAL OF MATHEMATICS
}

\author{
Founded in 1951 by
}

E. F. Beckenbach (1906-1982) $\quad$ F. Wolf (1904-1989)

\section{EDITORS}

Sun-Yung A. Chang (Managing Editor)

University of California

Los Angeles, CA 90095-1555

pacific@math.ucla.edu

F. Michael Christ

University of California

Los Angeles, CA 90095-1555

christ@math.ucla.edu

Nicholas Ercolani

University of Arizona

Tucson, AZ 85721

ercolani@math.arizona.edu
Robert Finn

Stanford University

Stanford, CA 94305

finn@gauss.stanford.edu

Steven Kerckhoff

Stanford University

Stanford, CA 94305

spk@gauss.stanford.edu

Martin Scharlemann

University of California

Santa Barbara, CA 93106

mgscharl@math.ucsb.edu
Gang Tian

Massachusettes Institute of Technology

Cambridge, MA 02139

tian@math.mit.edu

\author{
V. S. Varadarajan \\ University of California \\ Los Angeles, CA 90095-1555 \\ vsv@math.ucla.edu \\ Dan Voiculescu \\ University of California \\ Berkeley, CA 94720 \\ dvv@math.berkeley.edu
}

\section{SUPPORTING INSTITUTIONS}

ACADEMIA SINICA, TAIPEI

CALIF. INST. OF TECHNOLOGY

CHINESE UNIV. OF HONG KONG

HONG KONG UNIV. OF SCI. \& TECH.

KEIO UNIVERSITY

MACQUARIE UNIVERSITY

MATH. SCI. RESEARCH INSTITUTE

NEW MEXICO STATE UNIV.

OREGON STATE UNIV.

PEKING UNIVERSITY

RITSUMEIKAN UNIVERSITY

STANFORD UNIVERSITY

$\begin{array}{ll}\text { TOKYO INSTITUTE OF TECHNOLOGY } & \text { UNIV. OF CALIF., SANTA CRUZ } \\ \text { UNIVERSIDAD DE LOS ANDES } & \text { UNIV. OF HAWAII } \\ \text { UNIV. OF ARIZONA } & \text { UNIV. OF MELBOURNE } \\ \text { UNIV. OF BRITISH COLUMBIA } & \text { UNIV. OF MONTANA } \\ \text { UNIV. OF CALIF., BERKELEY } & \text { UNIV. NACIONAL AUTONOMA DE MEXICO } \\ \text { UNIV. OF CALIF., DAVIS } & \text { UNIV. OF NEVADA, RENO } \\ \text { UNIV. OF CALIF., IRVINE } & \text { UNIV. OF OREGON } \\ \text { UNIV. OF CALIF., LOS ANGELES } & \text { UNIV. OF SOUTHERN CALIFORNIA } \\ \text { UNIV. OF CALIF., RIVERSIDE } & \text { UNIV. OF UTAH } \\ \text { UNIV. OF CALIF., SAN DIEGO } & \text { UNIV. OF WASHINGTON } \\ \text { UNIV. OF CALIF., SANTA BARBARA } & \text { WASHINGTON STATE UNIVERSITY }\end{array}$

The supporting Institutions listed above contribute to the cost of publication of this Journal, but they are not owners or publishers and have no responsibility for its contents or policies.

\section{Manuscripts must be prepared in accordance with the instructions provided on the inside back cover.}

The table of contents and the abstracts of the papers in the current issue, as well as other information about the Pacific Journal of Mathematics, may be found on the Internet at http://www.math.uci.edu/pjm.html.

The Pacific Journal of Mathematics (ISSN 0030-8730) is published monthly except for July and August. Regular subscription rate: $\$ 245.00$ a year (10 issues). Special rate: $\$ 123.00$ a year to individual members of supporting institutions.

Subscriptions, back issues published within the last three years and changes of subscribers address should be sent to Pacific Journal of Mathematics, P.O. Box 4163, Berkeley, CA 94704-0163, U.S.A. Prior back issues are obtainable from Kraus Periodicals Co., Route 100, Millwood, NY 10546.

The Pacific Journal of Mathematics at the University of California, c/o Department of Mathematics, 981 Evans Hall, Berkeley, CA 94720 (ISSN 0030-8730) is published monthly except for July and August. Second-class postage paid at Berkeley, CA 94704, and additional mailing offices. POSTMASTER: send address changes to Pacific Journal of Mathematics, P.O. Box 6143, Berkeley, CA 94704-0163.

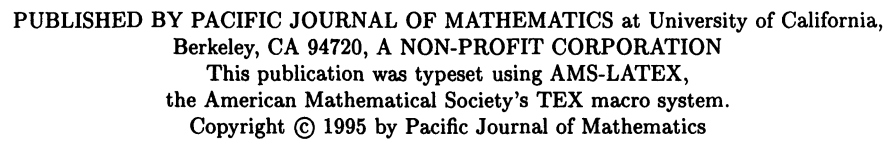




\title{
PACIFIC JOURNAL OF MATHEMATICS
}

\author{
Volume $176 \quad$ No. $1 \quad$ November 1996
}

Moduli spaces of isometric pluriharmonic immersions of Kähler manifolds into

indefinite Euclidean spaces

HitOSHI FURUHATA

On a theorem of Koch

FARSHID HAJIR

Degree-one maps onto lens spaces

Claude Hayat-Legrand, Shicheng WANG and Heiner Zieschang

Unitary representation induced from maximal parabolic subgroups for split $F_{4}$

CHENG CHON Hu

New constructions of models for link invariants

FRANÇOIS JAEGER

Solvability of Dirichlet problems for semilinear elliptic equations on certain domains

ZHIREN JIN

Hadamard-Frankel type theorems for manifolds with partially positive curvature

Katsuei KenMotsu and CHANGYU XIA

Boundary behavior of the Bergman curvature in strictly pseudoconvex polyhedral domains

KANG-TAE KIM and JIYE YU

Existence and behavior of the radial limits of a bounded capillary surface at a corner

KIRK LANCASTER and DAVID SIEGEL

Triangle subgroups of hyperbolic tetrahedral groups

COLIN MACLACHLAN

Chern classes of vector bundles on arithmetic varieties

TOHRU NAKASHIMA and YUICHIRO TAKEDA

Haar measure on $E_{q}(2)$

ARUP KUMAR PAL

Domains of partial attraction in noncommutative probability

VITTORINO PATA

Partitioning products of $\mathscr{P}(\omega) /$ fin

OTMAR SPINAS

Dimensions of nilpotent algebras over fields of prime characteristic

CORA M. STACK

Tensor products of structures with interpolation

FRIEDRICH WEHRUNG

Fourier multipliers for $L_{p}\left(\mathbb{R}^{n}\right)$ via $q$-variation 